\title{
PROGRAMA MAIS MÉDICOS EM MUNICÍPIO DE FRONTEIRA INTERNACIONAL E OS DESAFIOS DA GESTÃO EM SAÚDE
}

\author{
"MORE DOCTORS" PROGRAM IN A MUNICIPALITY AT AN INTERNATIONAL \\ BORDER AND THE CHALLENGES OF MANAGEMENT IN HEALTH
}

\author{
Carlos Guilherme Meister Arenhart ${ }^{1}$ iD)(0000-0003-1937-2050), Ludmila Mourão Xavier Gomes ${ }^{2}$ iD) (0000- \\ 0001-6442-5719), Elisete Maria Ribeiro², Thiago Luis de Andrade Barbosa² (DD) (0000-0002-6985-9548) \\ ${ }^{1}$ Universidade Estadual do Oeste do Paraná, Foz do Iguaçu, Paraná, Brasil. \\ <carlosmeistera@gmail.com> \\ ${ }^{2}$ Universidade Federal de Integração Latino-Americana, Foz do Iguaçu, Paraná, Brasil.
}

Resumo A cooperação técnica entre os países latinoamericanos tem promovido grandes debates no campo da saúde coletiva e na consolidação da gestão do trabalho no Sistema Único de Saúde. Objetivou-se compreender as concepções de gestores da Atenção Primária à Saúde de um município em região de fronteira - historicamente distante do movimento da reforma sanitária brasileira sobre a implantação do Projeto Mais Médicos. Trata-se de um estudo qualitativo, fundamentado na hermenêuticadialética, de abordagem exploratória, realizado na região de fronteira trinacional entre Brasil, Paraguai e Argentina. Foram realizadas entrevistas abertas, gravadas, transcritas e analisadas de forma interpretativa e dialética no âmbito empírico-conceitual. No entendimento das gestoras, o Projeto contribuiu para a manutenção da oferta de serviços na Atenção Primária local, e é um caminho para a garantia de prestação da assistência médica em município de região fronteiriça. O Projeto também colaborou para a corresponsabilização pelo cuidado. Observou-se movimento dialético no atributo longitudinalidade e também nas dificuldades de operacionalizar o binômio gestão-planejamento, demonstrando contradição nas práxis operacionais dos serviços. Conclui-se que, para as gestoras entrevistadas, o Projeto mostrou-se importante estratégia de gestão do trabalho em saúde, conquanto haja um nó crítico baseado no pontual período de permanência destes profissionais nas equipes da Atenção Primária.

Palavras-chave cooperação internacional; organização e administração; atenção primária à saúde.
Abstract The technical cooperation among Latin American countries has been promoting great debates in the field of collective health and in the consolidation of labor management in the Brazilian Unified Health System (SUS, in the Portuguese acronym). The present paper had the goal of understanding the conceptions of Primary Health Care managers in a municipality located in a border region - which has been historically distant from the Brazilian sanitary reform movement - regarding the implementation of the "More Doctors" Program. The study is of a qualitative nature, and it was based on the hermeneutic-dialectic method, with an exploratory approach, and was conducted in the tri-border area of Brazil, Paraguay and Argentina. We performed open interviews that were recorded, transcribed and analyzed in an interpretive and dialectic manner in the empirical-conceptual context. In the understanding of the managers, the Program contributed to the continuance of the offer of services in the local Primary Health Care, and it is a way to ensure the provision of medical care in a municipality located in a border region. The Program also contributed to the co-responsibilization for the care. We observed a dialectic movement in the longitudinality attribute as well as in the difficulties to operationalize the binomial management-planning, demonstrating a contradiction in the operational praxis of the services. We concluded that, for the managers interviewed, the Program was an important strategy for the management of labor in health, even though there is a critical issue regarding the fleeting period these professionals remain in the Primary Health Care teams.

Keywords international cooperation; organization and administration; primary health care. 


\section{Introdução}

As orientações para Atenção Primária à Saúde (APS) discutidas no Comitê Regional para as Américas da World Health Organization (WHO) e aprovadas na Declaração de Montevidéu ainda possuem grandes desafios para sua consolidação. Os países latino-americanos comprometeram-se a implementar modelos de atenção centrados em pessoas e comunidades. Contudo, as medidas propostas são prejudicadas pelas realidades e práticas vigentes (Stigler, 2016; Organização Pan-Americana da Saúde, 2005, 2017).

Apesar de a Declaração de Alma-Ata ter promovido, nos países latinoamericanos, debates e a construção de estratégias para consolidação da APS como eixo coordenador dos sistemas de saúde, o que imperou nesses países, após a referida conferência, foi a implantação de uma APS seletiva (Giovanella e Almeida, 2017; Pires-Alves e Cueto, 2017).

A conferência de Alma-Ata proporcionou o entendimento de que a APS se tornasse essencial e fundada em tecnologias apropriadas e custoefetivas nos sistemas de saúde. Desse modo, a gestão em saúde nos espaços de tomada de decisão é um importante fator a ser considerado no estabelecimento das práxis e princípios da APS e, mais recentemente, a cooperação internacional surge como estratégia de gestão do trabalho no Brasil (OPAS, 2017; Turci, Lima-Costa e Macinko, 2015).

O Programa Mais Médicos para o Brasil é o mais recente acordo de cooperação técnica em saúde, lançado em 2013 pela lei n. 12.871, que materializou a cooperação entre Brasil, Organização Pan-Americana da Saúde e Cuba. Dentre seus oito principais objetivos, destaca-se a necessidade de se diminuir a carência de médicos nas regiões prioritárias para o Sistema Único de Saúde (SUS), a fim de reduzir as desigualdades regionais na área da saúde e fortalecer a prestação de serviços da APS. É neste programa que se encontra o delineamento do Projeto Mais Médicos (PMM), que visa fortalecer o provimento de profissionais na rede de cuidados primários (Oliveira et al., 2015; Brasil, 2017).

As concepções e práxis de gestores da APS são fundamentais para a implantação efetiva do PMM, considerando a indissociabilidade da atençãoeducação-gestão para a consolidação das políticas de saúde nos municípios. Em janeiro de 2018, existiam cerca de 18.240 profissionais médicos vinculados ao programa, distribuídos em 4.058 municípios. Tais profissionais atenderam à cerca de 63 milhões de brasileiros que, antes desta iniciativa, não possuíam acesso à APS (Brasil, 2017; Oliveira et al., 2015). Entretanto, em meados de dezembro de 2018, houve grandes rupturas na operacionalização do projeto e Cuba anunciou a retirada de seus profissionais do país, deixando os municípios desassistidos.

O presente estudo buscou compreender as concepções de gestores da APS em região de fronteira trinacional entre Brasil, Paraguai e Argentina sobre a 
cooperação internacional em saúde, baseando-se no processo de implantação do PMM.

\section{Metodologia}

Este estudo é qualitativo e fundamentado na hermenêutica-dialética, que consiste na afirmação extrema do significado prático da razão humana. A escolha dos dois métodos não foi simplesmente por terem a práxis como objeto, mas porque não há práxis no seu sentido pleno sem que se pressuponha os horizontes do pensamento dialético-hermenêutico (Minayo, 2002).

Entre as diferenças e as mediações possíveis, a união destes dois métodos permitiu a compreensão dos resultados como frutos de múltiplas determinações, apresentando-se como uma realidade que se mostra e se esconde na comunicação em saúde coletiva. Os consensos coexistem, simultaneamente, entre tensões e contradições (Minayo, 2002).

O cenário de realização do estudo foi o município de Foz do Iguaçu, região de fronteira trinacional entre Brasil, Paraguai e Argentina no estado do Paraná e possui uma população aproximada de 260 mil habitantes.

A APS municipal é articulada por uma rede de saúde com 27 unidades básicas de saúde (UBSs) e 32 equipes da Estratégia Saúde da Família (ESF), organizadas, territorialmente, em cinco distritos sanitários (DSs) e 26 médicos do PMM, sendo nove deles cubanos. A singularidade da APS por estar em região de fronteira decorre da existência de atendimento a estrangeiros, paraguaios, argentinos, brasiguaios e também da tímida produção científica sobre a APS.

A pesquisa foi realizada com profissionais que atuam como gestores de saúde no âmbito da APS na gestão central, na gestão dos DSs e no microespaço da gestão das UBSs. Nesta pesquisa, o encerramento da amostra ocorreu pelo princípio da saturação teórico-discursiva. Tal princípio é definido como a suspensão de inclusão de novos participantes quando os dados obtidos passam a apresentar redundância e repetição (Fontanella et al., 2011).

A coleta de dados ocorreu entre maio e setembro de 2017, com entrevistas abertas de duração aproximada de 60 minutos com 15 gestores, por meio da pergunta norteadora: O que o Projeto Mais Médicos representa para você?

A análise dos dados ocorreu de forma interpretativa, utilizando-se dos pressupostos epistemológicos da corrente hermenêutica-dialética. A operacionalização ocorreu de acordo com a elaboração de categorias analíticas; ordenação e classificação dos dados; leitura horizontal e transversal das entrevistas e análise final (Minayo, 2002). Ressalta-se que a hermenêutica-dialética não dispõe de técnicas que devam ser seguidas a rigor, porém a etapa de análise dos dados é tida como um caminho do pensamento (Minayo, 2002). Para trilhar esse caminho, os pesquisadores percorreram os seguintes passos: 
análise do contexto histórico, encontro com a realidade, sistematização das interpretações, categorização e análise dos consensos e dissensos, conforme ilustrado na Figura 1.

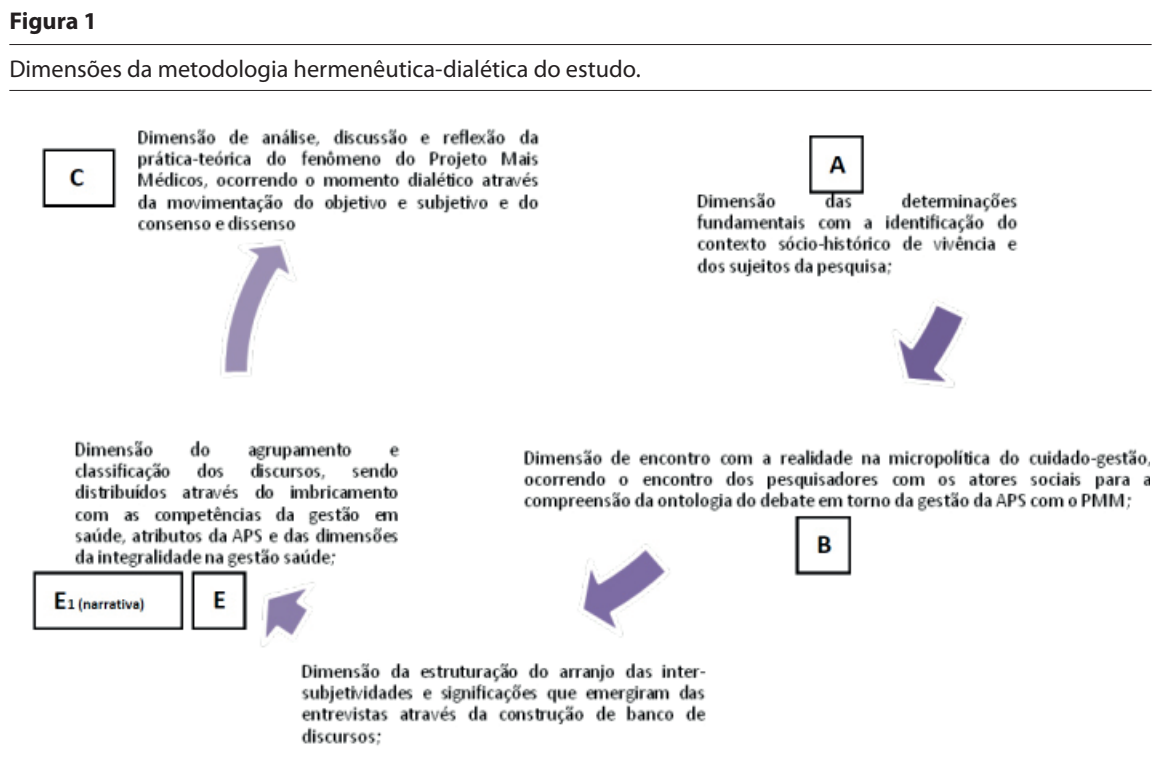

Fonte: Elaborada pelos autores

Para manter o anonimato, as falas das entrevistadas foram codificadas pela letra E seguida de um número em arábico sequencial.

A pesquisa foi aprovada pelo Comitê de Ética em Pesquisa da Faculdade Assis Gurgacz, Paraná, sob o registro CAAE de nº 65642017300005219 e respeitou todos os princípios éticos. Os participantes assinaram o termo de consentimento livre e esclarecido.

\section{Resultados e discussão}

Os gestores da região trinacional são mulheres com idade acima de 35 anos. A formação das gestoras é de níveis médio e técnico, sendo que apenas três possuem escolaridade de nível superior no provimento de seu cargo. A área de formação e trabalho das gestoras é, principalmente, a enfermagem. O tempo médio de atuação e trabalho na aderência em gestão de saúde é de 13,5 anos.

A força de trabalho em saúde coletiva na APS dos países latino-americanos é considerada um fator crítico de sucesso dos sistemas locais de saúde e tem sido evidenciado em estudos recentes. A disponibilidade, a formação e os vínculos dos profissionais representam um dos grandes desafios para a implementação dos novos modelos (Giovanella e Almeida, 2017). 
As subjetividades nos processos de trabalho da gestão da atenção primária à saúde municipal, as falas das gestoras sobre o PMM e a interlocução das distâncias e proximidades discursivas sobre a cooperação internacional em saúde foram agrupadas em categorias que foram discutidas à luz dos pressupostos da atenção primária à saúde e do SUS. Com base na análise de dados, a interpretação da categoria temática que emergiu foi agrupada em três subcategorias relacionadas ao PMM: 1) os sentidos atribuídos à inserção do Projeto Mais Médicos no contexto de região fronteiriça; 2) o PMM na longitudinalidade da APS e as interfaces do trabalho da gestão; 3) o trabalho humanizado dos médicos do PMM.

\section{Os sentidos atribuídos ao Projeto Mais Médicos no contexto de região fronteiriça}

O PMM emerge de uma necessidade sócio-sanitária de interiorizar a disponibilidade de profissionais médicos na APS do Brasil. No cenário municipal, situado em região fronteiriça, a compreensão dos gestores é de que o PMM tem representado uma possibilidade para o funcionamento integral dos serviços de APS.

Antes da implantação do PMM, a APS foi caracterizada nos discursos como complexa e com direção de calamidade. A reflexão das gestoras enuncia importante valorização do programa para a prevenção de um possível 'caos' na prestação dos serviços de saúde. O PMM é interpretado como caminho para garantia de prestação da assistência:

O projeto chegou justo nestes quatros anos que eu achei que toda a APS ia por água abaixo e daí agarrei com todas as forças. (E01)

A vinda dos médicos pra cá veio cobrir um vazio assistencial na fronteira. Abriam concursos e não completava as vagas nunca. Depois do programa não falta mais médicos na atenção básica. (E08)

A metáfora 'por água abaixo', utilizada na linguagem da entrevistada, aponta que os sentidos atribuídos ao contexto direcionam para a atomização da rede de cuidados básicos. A APS apresentava deficiência no provimento de profissionais médicos.

A expressão 'cobrir um vazio assistencial' legitima a resolutividade do programa, em que as tentativas de desatar o nó crítico do provimento de profissionais já estava chegando a seu esgotamento na cidade.

O impacto do sentido de necessidade e possibilidade atribuída pelas gestoras refere que esta estratégia de cooperação técnica ocasionou importante direcionamento na APS em município de região fronteiriça, considerado como historicamente distante das políticas públicas em saúde articuladas no Brasil. 
Estudo recente analisou o índice de escassez de médicos na APS entre 2013 e 2015 em municípios brasileiros para mensurar a redução das desigualdades no acesso à APS durante a implantação do PMM. Dentre os resultados, destacase a insegurança assistencial decorrente da substituição da oferta regular municipal pelo provimento federal. O PMM pode ter fortalecido a dependência de alguns municípios à gestão federal do SUS, especialmente aqueles que, no contexto sócio-histórico, apresentavam carência e dificuldades no provimento de profissionais médicos na APS (Girardi et al., 2016). No cenário deste estudo em região fronteiriça, verifica-se a dependência do município à gestão federal do SUS por meio do PMM para manutenção da APS, em detrimento da dependência da categoria profissional médica existente anteriormente.

Os municípios pequenos eram reféns. O médico vinha com uma exigência salarial, quase que ele recebia o total de uma equipe toda. Perdia-se um profissional com muita facilidade porque o município vizinho oferecia mil reais a mais e eles iam. (E06)

Eu tinha muito problema. Aqui é uma área de alta vulnerabilidade e os médicos não queriam estar aqui. (E03)

As inquietudes expressas mostram a preocupação da gestão em saúde com a APS e os impactos da precariedade de oferta da força de trabalho médica, principalmente em unidades básicas de saúde de territórios de alta complexidade social.

Sobre a dificuldade de fixação de médico apontada neste estudo, destacase que esta não é só uma realidade brasileira. Países desenvolvidos como Austrália (Clark et al., 2013) e Estados Unidos (WHO, 2013) também enfrentaram problemas de provisão e de fixação desses profissionais.

Apesar da realização de concursos públicos na região fronteiriça, não havia o provimento e ocupação nos cargos. O sentido atribuído da metáfora 'eram reféns' demonstra a contradição entre a operacionalização das competências da gestão em saúde, especialmente aquelas imbricadas com a gestão do trabalho, planejamento e a avaliação em saúde sobre a categoria médica.

Os conflitos no âmbito da gestão em saúde intermunicipal para fixar os profissionais médicos entre os municípios da mesma região de saúde estão nos discursos, demonstrando o condicionamento dúbio entre o capital financeiro e a força de trabalho médica na rede de atenção à saúde. A dificuldade na provisão e fixação de médicos é uma realidade em muitos municípios brasileiros. Tal problemática advém dos prejuízos causados pelos contextos de heteronomia salarial, jornada de trabalho diferenciada e desigual, inexistência de planos de cargos, carreira e salários, ausência de 
diretrizes e princípios técnico-institucionais no processo de contratação por clientelismo (Silva et al., 2016).

As gestoras também evidenciaram as iniquidades sociais dos territórios sob responsabilidade das equipes da APS e a dificuldade de fixação de médicos em territórios vulneráveis econômica e socialmente. É fato que condições inadequadas de trabalho, cargas horárias excessivas e má remuneração dificultam a fixação de médicos (Silva et al., 2016; Silva et al., 2017).

O problema do gestor, nesta pesquisa, é representado na adversidade de lotação dos profissionais médicos em regiões vulneráveis e o PMM foi a possibilidade de planejamento da distribuição dos novos médicos para a APS segundo critérios de equidade, vulnerabilidade social e de populações específicas (Silva et al., 2017; Campos e Pereira, 2016).

Pesquisa brasileira aponta a significação polissêmica nos impactos do PMM no âmbito da territorialidade em saúde. Ações e práticas em curto prazo são privilegiadas com o provimento de médicos e, por possuir aderência a territórios marginalizados historicamente, permanece ainda como empecilho no estabelecimento de relações com estruturas locais já existentes (Lotta, Pinto e Silva, 2017). Ressalte-se que fatores estruturais e organizacionais são importantes para o desempenho da APS e provimento de profissionais médicos, o que requer importantes investimentos nas estruturas municipais de saúde (Turci, Lima-Costa e Macinko, 2015). A conjuntura municipal e a plataforma social entre o âmbito público e privado são apresentadas como dificuldade no provimento dos profissionais na rede de atenção à saúde:

Os médicos têm muita oferta no mercado privado e eles não param na APS. (E09)

A 'oferta' é compreendida como a disponibilidade de salários maiores no âmbito da atenção privada, o que pode tornar os médicos transitórios na APS. O trabalho na rede privada representa oportunidades de ganhos salariais superiores à rede pública, fazendo com que haja preferências por estes serviços. Percebe-se que essa realidade não é algo exclusivo da região fronteiriça, o que se repete em outras localidades, como é apontado em outros estudos (Feitosa-Filho et al., 2012; Watte et al., 2015).

Nesta pesquisa, evidenciou-se que o PMM possibilitou, momentaneamente, a manutenção da gestão do trabalho em saúde da APS e a oferta de serviços médicos no SUS em região fronteiriça, mas há questões de continuidade e descontinuidade dos profissionais do PMM que também são discutidas pelas gestoras. 


\section{A continuidade e a descontinuidade do PMM e as interfaces do trabalho da gestão}

A rede de significados revela que o PMM fortaleceu o vínculo de cuidado entre profissionais e usuários dos serviços na APS. Um movimento dialético surgiu, pois, nas narrativas, constatou-se que, na área de gestão e planejamento, o atributo longitudinalidade da APS foi uma contradição, visto que o contrato dos médicos se encerrou em três anos.

Segundo as gestoras, o PMM aborda um dos atributos da APS, caracterizado pela continuidade do cuidado nos diferentes ciclos de vida da população e apontado como atributo de uma APS resolutiva.

É muito importante esse programa, porque trouxe a possibilidade de atender um dos princípios da APS, que é a longitudinalidade, e com nosso quadro não dava. (E09)

Para esse movimento hermenêutico-dialético, de polissemia dos conceitos da longitudinalidade no cuidado em saúde, utilizamos como base epistemológica a definição de longitudinalidade como relação entre profissionais de saúde e usuários, transcendendo diversos episódios de saúde e doença e fortalecendo o vínculo de confiança e responsabilidade (Cunha e Giovanella, 2011).

Investigação recente realizada no Paraná, Brasil (Carrer et al., 2016), com o intuito de verificar os atributos da APS em UBS, com e sem profissionais do PMM, evidenciou o movimento contraditório e dialético no atributo longitudinalidade. Como resultado, elucida o debate sobre o impacto neste atributo, que é pontual no cuidado em saúde, semelhante aos profissionais do PMM neste estudo. Esta é uma importante preocupação, tendo em vista que é um atributo considerado central do modelo brasileiro de APS (Flôr et al., 2017).

Considera-se adequada a caracterização do atributo longitudinalidade, com base na requisição de apropriação territorial, vínculo e conhecimento do modo de viver das comunidades locais, sobre as práticas de produção de saúde e iatrogênicas, os caminhos e métodos que os sujeitos utilizam para a promoção da vida e da felicidade (Brasil, 2009). Neste caso, a permanência pontual destes profissionais nas equipes do PMM não permite o cuidado longitudinal, mas amplia o acesso aos serviços ofertados.

A pontual permanência dos profissionais nas equipes da APS poderia ser corrigida por uma 'nova' configuração legal do projeto proposta pelos gestores:

Como gerente, eu acho que, para aqueles médicos que querem permanecer no Brasil, tinha que liberar o CRM. Porque eles criam vínculo e vão embora. Os nossos foram embora, arrasados. Já tinham se enraizado aqui. (E01) 
Depois que terminou o contrato da nossa primeira médica foi muito ruim, porque não tinha médico com a gente aqui. (E13)

O movimento dialético pode ser potencializado na análise, considerado o contexto do discurso e a amplitude da significação do vínculo do cuidado. O enraizamento citado na concepção da gestora refere-se ao vínculo com a equipe e os usuários no ato do cuidado. Consiste na estruturação das práticas clínicas dos cuidados oferecidos na UBS e na prática entre os trabalhadores das equipes.

O impedimento da permanência dos profissionais nas equipes é interpretado como processo burocrático qualitativamente prejudicial para o vínculo constituído e para a longitudinalidade nas ações e serviços desenvolvidos por esses profissionais na cidade. O rompimento e o retorno destes profissionais aos seus países, o que já estava previsto no contrato bilateral, impactou negativamente na lógica gerencial, considerando a desassistência ocorrida nas equipes e a descontinuidade dos serviços ofertados.

Compreende-se que um dos entraves históricos na APS da região fronteiriça atribuída pelas gestoras é a ausência de profissionalização na tomada de decisão em saúde, o imediatismo nos processos de trabalho e a característica centralizadora dos espaços de gestão, como se pode obsevar no relato a seguir:

\footnotetext{
A gente trabalha no limite do limite e qualquer imprevisto vira um drama. A APS tem que ser bem observada pelo gestor e prefeito. A pressão chega do ministério para você implantar determinados serviços e ela não aguarda os tempos públicos para você agir e ele quer pra ontem e você tem que buscar em algum lugar e quando acaba o contrato do PMM você não consegue agir (E08).
}

Uma reflexão dialética, anteriormente oculta, é evidenciada na interface entre o movimento da gestão do SUS nacional e a necessidade de rápido planejamento local para sua execução no microespaço da política municipal.

O termo 'limite do limite' é postulado como atributo local da identidade do ato de planejar na gestão da APS municipal. Essa fala demonstra a dificuldade de realizar um planejamento estratégico local e esclarece que a gestão em saúde coletiva está pautada no modelo biomédico. Centra-se nos problemas imediatos que surgem no cotidiano do trabalho e não prioriza a promoção e prevenção em saúde - destacadas em Alma-Ata e postuladas como práticas reordenadoras dos sistemas de saúde.

Além da continuidade e descontinuidade dos serviços, há um destaque para o trabalho humanizado realizado pelos profissionais do PMM. 


\section{O trabalho humanizado dos médicos do PMM}

O trabalho na APS apresenta uma complexidade que envolve cooperação e humanização nas relações. A humanização propõe novas abordagens na relação e encontros entre trabalhadores, gestores e usuários do SUS. A produção de afeto na relação clínica, o diálogo horizontal, o compromisso e a autonomia na produção de vida são importantes destaques da estratégia ao reorientar a gestão do cuidado na APS (Brasil, 2009).

Há uma compreensão que elucida possíveis diferenças nas práticas clínicas, no relacionamento entre profissionais e coletivo com a equipe de saúde, na abordagem aos usuários e nos impulsos na resolução de entraves assistenciais com médicos do PMM.

Eles [os usuários] estão acostumados com trato mais frio e mecanizado, tipo robozinho e esses profissionais não são assim. Eles [médicos do PMM] têm empatia e tratam com mais zelo o usuário. A cultura e o amor muda tudo. (E09)

Esse médico vem com uma humanização à flor da pele. Com uma visão e respeito à equipe muito clara, diferente do nosso formado aqui, que demora a se habituar à necessidade da equipe. (E04)

Os valores compartilhados na cultura institucional que prevalece nos serviços de saúde ainda são a fragmentação, as relações hierárquicas de trabalho, a atuação profissional individualizada e o paroxismo instrumental da racionalidade técnico-científica (Peduzzi, 2011). Os sentidos atribuídos pelas gestoras pressupõem que isso é diferente no contexto do PMM. Os encontros entre o médico do PMM e os usuários têm tensionado as práticas e concepções de cuidados na APS. O modo de gestão da clínica, pautado na redução de distanciamento entre o trabalhador e médico-usuário e entre médico do PMM e equipes, denota o direcionamento biopsicossocial ao modo de produzir vida vigente até então na APS.

A relação socioafetiva e de confiança é uma diretriz importante na atuação destes profissionais e está presente nos discursos das gestoras quando discutem uma potencialização na orientação das condutas trazidas por esses trabalhadores para a APS.

Eu acho que a humanização é a potência. Ter amor ao cuidado. Eu senti muito e a população elogia. Eles têm muita calma para explicar, falam devagarinho pro usuário entender. (E08)

A responsabilização do caminhar do usuário pela rede, corresponsabilização é um destaque. Cubanos que vão ao serviço hospitalar visitar o usuário quando eles vão 
internar, fazer uma cirurgia. Corresponsabilidade total. Para a grande maioria, a língua não foi dificultadora. Eles se encontraram rápido aqui. (E10)

As percepções sobre a epistemologia prática de humanização dos profissionais do PMM introduz no debate um cenário de harmonização para o cumprimento da competência da gestão em saúde caracterizada pela responsabilidade sanitária das equipes com a comunidade.

Compreendemos que uma das grandes dificuldades que ainda permeiam as práticas na APS é a incorporação de tecnologias leves, principalmente aquelas ligadas ao encontro comunicacional entre trabalhador e usuários. A orientação formativa desses profissionais é diferenciada e pode ser evidenciada pela expressão 'calma ao explicar' e pela necessidade de o 'usuário entender' a conduta médica.

Um rompimento de paradigma socialmente constituído, quando o PMM foi implantado, é legitimado pelos usuários, segundo as falas dos gestores. O idioma não impede o desenvolvimento das atividades. $\mathrm{O}$ contexto da multiculturalidade, que é uma característica da região fronteiriça do estudo, e a vivência cotidiana da língua portuguesa e espanhola facilitaram a inserção desses profissionais.

O comprometimento com os usuários é interpretado como corresponsabilidade do cuidado, fortalecendo a lógica da APS como coordenadora do cuidado. Estudo realizado em Mossoró, município do Rio Grande do Norte Brasil, evidenciou as percepções dos usuários sobre o PMM na lógica biopsicossocial do ato clínico e a potencialidade da prática humanizada em saúde com a corresponsabilização (Silva et al., 2016). Estes resultados aproximam-se dos movimentos hermenêuticos das falas das gestoras do presente estudo.

Uma subjetividade importante é a atuação compromissada com as necessidades dos usuários vinculados às equipes. Contrapondo a prática de médicos não vinculados ao PMM, a gestora local faz a indução analítica sobre a eficiência do trabalho desses profissionais na rede de cuidados primários.

E sinto amor à camisa por parte deles. Eles não põem tantos obstáculos e não colocam dificuldade. Fazem milagres aqui e o negócio deles é resolver o problema do usuário. (E14)

O jeito deles trabalharem e abordarem os usuários é até superior à nossa medicina brasileira. (E05)

As expressões 'obstáculos' e 'dificuldades' denotam a interpretação da atuação com foco na resolução das necessidades dos usuários. Estas expressões articulam-se dialeticamente com a alegoria 'superior', elucidando que, na 
ótica gerencial da APS, faz-se necessário, também, conduzir o ato de cuidado balizado nas tecnologias leves em detrimento das tecnologias duras impostas pelo modelo hegemônico vigente. A atribuição de qualidade sobre a prática dos profissionais é referenciada nas generalidades sociotécnicas da conjuntura da APS, demonstrando as dificuldades da gestão da APS em operacionalizar novas condutas na gestão do cuidado.

As limitações do presente estudo são aquelas relacionadas aos estudos qualitativos. O número de atores entrevistados e o fato do estudo ter sido realizado apenas com gestores que são da categoria profissional da enfermagem e o contexto municipal são limitações do estudo.

\section{Conclusão}

No âmbito da gestão em saúde, permanecem movimentos tensionadores sobre as concepções da APS. A compreensão das gestoras sobre o impacto do PMM na APS de região trinacional elucida a importância deste programa para o fortalecimento da APS e há uma valorização positiva e simbólica dos gestores sobre o projeto. Se anteriormente a dependência organizacional municipal estava circunscrita ao corporativismo médico, hoje é evidenciada a dependência da esfera federal de gestão.

Ressalta-se uma provável fragilidade na compreensão sobre a longitudinalidade do cuidado. A continuidade ocorrida com a provisão de médico e a descontinuidade com o término do contrato do PMM interfere na implantação do planejamento situacional e estratégico no âmbito da gestão da atenção básica municipal.

A cooperação técnica em saúde em região fronteiriça na América Latina pode ser importante estratégia para fortalecer os debates em torno do trabalho na APS e suas fragilidades ainda permanentes após 40 anos de Alma-Ata. As contribuições desta pesquisa podem potencializar estratégias locais da APS em região de fronteira, especialmente no direcionamento da cooperação em saúde como tecnologia para qualificação da saúde pública. 


\section{Colaboradores}

Carlos Gulherme Meister Arenhart contribuiu no projeto do trabalho, aquisição, análise e interpretação de dados, bem como a redação do artigo e aprovação final da versão a ser publicada. Ludmila Mourão Xavier Gomes contribuiu com a concepção do trabalho, análise, interpretação dos dados e revisão crítica do conteúdo intelectual; Elisete Maria Ribeiro contribuiu com o projeto do trabalho; Thiago Luis de Andrade Barbosa realizou revisão crítica do conteúdo intelectual.

\section{Financiamento}

Ministério da Educação, Programa de Residência Multiprofissional em Saúde da Família.

\section{PROGRAMA MÁS MÉDICOS EN UNA CIUDAD DE FRONTERA IN- TERNACIONAL Y LOS DESAFÍOS DE LA GESTIÓN DE LA SALUD}

Resumen La cooperación técnica entre los países latinoamericanos ha promovido grandes debates en el ámbito de la salud pública y en la consolidación de la gestión del trabajo en el Sistema Único de Salud Brasileño. El objetivo fue comprender los puntos de vista de gestores de la Atención Primaria de la Salud de una ciudad fronteriza - históricamente alejada del movimiento de la reforma sanitaria brasileña - sobre la implantación del Proyecto Más Médicos. Se trata de un estudio cualitativo, fundamentado en la hermenéutica dialéctica, de abordaje exploratorio, realizado en la región de la triple frontera entre Brasil, Paraguay y Argentina. Se realizaron entrevistas abiertas, grabadas, transcritas y analizadas de forma interpretativa y dialéctica en el ámbito empírico-conceptual. En la opinión de las gestoras, el Proyecto contribuyó para mantener el suministro de servicios en la Atención Primaria local y es una manera de garantizar la prestación de la asistencia médica en ciudades fronterizas. Así mismo, el Proyecto colaboró para la corresponsabilidad por el cuidado. Se observó un movimiento dialéctico en el atributo longitudinalidad así como en las dificultades de implementar el binomio gestión-planificación, demostrando una contradicción en las prácticas operativas de los servicios. Se concluye que para las gestoras entrevistadas, el Proyecto fue una estrategia importante de gestión del trabajo en salud, más allá que se observe un nudo crítico en el período puntual de permanencia de estos profesionales en los equipos de Atención Primaria. Palabras clave cooperación internacional; organización y administración; atención primaria de la salud. 


\section{Referências}

BRASIL. Ministério da Saúde. Programa mais médicos para o Brasil. Brasília: Ministério da Saúde, 2017.

BRASIL. Ministério da Saúde. Secretaria de Atenção à Saúde. Política nacional de humanização da saúde: gestão participativa e cogestão. Brasília: Ministério da Saúde, 2009.

CAMPOS, Gastão W. S.; PEREIRA JUNIOR, Nilton. A atenção primária e o programa mais médicos do sistema único de saúde: conquistas e limites. Ciência \& Saúde Coletiva, Rio de Janeiro, v.21, n.9, p. 2.6552.663, set. 2016 .

CARRER, Andreia et al. Efetividade da Estratégia Saúde da Família em unidades com e sem Programa Mais Médicos em município no oeste do Paraná, Brasil. Ciência \& Saúde Coletiva, Rio de Janeiro, v. 21, n. 9, p. 2.8492.860, set. 2016 .

CLARKE, Tyler R. et al. Medical graduates becoming rural doctors: rural background versus extended rural placement. The Medical Journal of Australia, Sydney, v.199, n. 11, p.779-782, 2013.

CUNHA, Elenice M.; GIOVANELLA, Ligia. Longitudinalidade/continuidade do cuidado: identificando dimensões e variáveis para a avaliação da Atenção Primária no contexto do sistema público de saúde brasileiro. Ciência \& Saúde Coletiva, Rio de Janeiro, v.16, supl. 1, p. 1.029-1.042, 2011.

FEITOSA-FILHO, Gilson S. et al. Reasons alleged by recently graduated physicians at Salvador/BA in 2010 to not attend the residency entrance exam. Revista Brasileira de Clínica Médica, São Paulo, v. 10, n. 2, p. 91-94. 2012.

FLÔR, Cristina R. et al. Primary health care as assessed by health professionals: comparison of the traditional model versus the Family Health Strategy. Revista Brasileira de Epidemiologia, São Paulo, v.20, n.4, p. 714-726, dez. 2017.
FONTANELLA, Bruno J. B. et al. Amostragem em pesquisas qualitativas: proposta de procedimentos para constatar saturação teórica. Cadernos de Saúde Pública, Rio de Janeiro, v. 27, n. 2, p. 389-394, fev. 2011.

GIOVANELLA, Ligia; ALMEIDA, Patty F. Comprehensive primary care and segmented health systems in South America. Cadernos de Saúde Pública, Rio de Janeiro, v. 33, supl. 2, 2017.

GIRARDI, Nicolau S. et al. Impacto do programa mais médicos na redução da escassez de médicos em atenção primária à saúde. Ciência \& Saúde Coletiva, Rio de Janeiro, v. 21, n. 9, p. 2.675-2.684, 2016.

LOTTA, Gabriela S. et al. Análise do programa mais médicos à luz dos arranjos institucionais: intersetorialidade, relações federativas, participação social e territorialidade. Ciência \& Saúde Coletiva, Rio de Janeiro, v. 21, n. 9, p. 2.761-2.772, 2016.

MACINKO, James et al. Gaps In Primary Care And Health System Performance In Six Latin American and Caribbean Countries, Health Affairs, Millwood, v. 35, n. 8, p. 1.513-1521, ago. 2016.

MINAYO, Maria C. S. Hermenêutica-dialética como caminho do pensamento social. In: MINAYO, Maria C. S.; DESLANDES, Suely F. Caminhos do pensamento: epistemologia e método. Rio de Janeiro: FIOCRUZ, 2002. p. 83-107.

OLIVEIRA, Felipe P. et al. Mais médicos: um programa brasileiro em uma perspectiva internacional. Interface: Comunicação, Saúde, Educação, Botucatu, v. 19, n. 54, p. 623-634, 2015.

ORGANIZAÇÃO PAN-AMERICANA DA SAÚDE. Declaração regional sobre as novas orientações para a atenção primária à saúde (Declaração de Montevidéu), $46^{\circ}$ Conselho Diretor, $57^{\text {a }}$ Sessão do Comitê Regional da OMS para as Américas, Washington, D.C., 26 a 30 de setembro de 2005 (CD46/13). 
ORGANIZAÇÃO PAN-AMERICANA DA SAÚDE. Saúde nas Américas, Edição de 2017. Resumo do panorama regional e perfil do Brasil. Washington, D.C.: OPAS, 2017.

PEDUZZI, Marina et al . Trabalho em equipe na perspectiva da gerência de serviços de saúde: instrumentos para a construção da prática interprofissional. Physis: Revista de Saúde Coletiva, Rio de Janeiro, v. 21, n. 2, p. 629-646, 2011.

PIRES-ALVES, Fernando A; CUETO, Marcos. The Alma-Ata decade: the crisis of development and international health. Ciência \& Saúde Coletiva, Rio de Janeiro, v. 22, n.7, jul. 2017.

SILVA, Oscarina E. et al. Geographical distribution of medical graduates from a public university. Revista da Associação Médica Brasileira, São Paulo, v. 63, n. 6, jun. 2017.

SILVA, Tiago R. B. et al. Percepção de usuários sobre o Programa Mais Médicos no município de Mossoró, Brasil. Ciência \& saúde coletiva, Rio de Janeiro, v. 21, n. 9, p. 2.861-2.869, 2016.
STARFIELD, Barbara. Primary Care: balancing health needs, services, and technology. Brasília: Ministério da Saúde, 2002.

STIGLER, Florian L. et al. No universal health coverage without primary health care. The Lancet, Londres, v. 387, n. 10.030, abr. 2016.

TURCI, Maria A.; LIMA-COSTA, Maria F.; MACINKO, James. Influência de fatores estruturais e organizacionais no desempenho da atenção primária à saúde em Belo Horizonte, Minas Gerais, Brasil, na avaliação de gestores e enfermeiros. Cadernos de Saúde Pública, Rio de Janeiro, v. 31, n. 9, p. 1.941-1.952, set.2015.

WATTE, Guilherme et al . Componentes Determinantes na Escolha da Especialização em Novos Profissionais Médicos. Revista Brasileira de Educação Médica, Rio de Janeiro, v. 39, n. 2, p. 193-195, jun. 2015

WORLD HEALTH ORGANIZATION (WHO). Health system in transition, United States of America: health system review, 2013. 